\title{
Properties of longitudinal flux tube waves
}

\section{Limiting shock strength behavior}

\author{
M. Cuntz ${ }^{1,2, \star}$ \\ ${ }^{1}$ Department of Physics, Science Hall, University of Texas at Arlington (UTA), Arlington, TX 76019-0059, USA \\ 2 Institute for Theoretical Astrophysics, University of Heidelberg, Tiergartenstraße 13, 69121 Heidelberg, Germany
}

Received 21 November 2003 / Accepted 16 March 2004

\begin{abstract}
We extend our previous work on analytic evaluations of properties of longitudinal tube waves to waves propagating in gravitational atmospheres. We derive an expression for the limiting shock strength and discuss the behavior of the shock strength in tubes of different geometry. It is found that a height-independent value for the limiting strength is attained for constant crosssection tubes and exponential tubes, whereas for wine-glass tubes the limiting shock strength increases with height due to the increase of the tube cross section. The limiting shock strength is well reproduced by time-dependent simulations. The derived limiting shock strength as well as the energy dissipation rate of the waves show significant similarities to acoustic waves. The limiting shock strength allows to estimate the heating potential of waves in the absence of detailed time-dependent computations.
\end{abstract}

Key words. magnetohydrodynamics (MHD) - shock waves - methods: analytical - Sun: chromosphere - stars: chromospheres

\section{Introduction}

Previous investigations have shown that longitudinal tube waves play an important role in the heating of chromospheres of different types of stars, including the Sun (e.g., Solanki 1996). Wave propagation in thin magnetic flux tubes has extensively been studied in the past (e.g., Roberts 1981, 1983; Spruit 1981a,b; Rae \& Roberts 1982; Edwin \& Roberts 1983) particularly in the case of negligible gravity and for the linear regime. In the work by Herbold et al. (1985) such a comparison has also been made for the non-linear regime. They found that longitudinal tube waves are similar to plane acoustic waves and particularly to acoustic tube waves. A main property of all three wave types is that they easily form shocks, which is the main reason why they are able to heat stellar atmospheres. Longitudinal tube waves and acoustic tube waves differ concerning the variation of the tube cross section. While for acoustic tube waves the tube cross section remains constant, the cross section of the magnetic tube waves varies during passage of longitudinal waves with the wave phase. It expands and contracts in a time-dependent manner while maintaining pressure balance with its surroundings. Model calculations based on longitudinal tube waves have been given for different types of stars (Narain \& Ulmschneider 1996, and references therein). Fawzy et al. (1998) presented models for the solar magnetic chromosphere assuming flux tubes of different spreading. The models indicate that the heating potential differs as function of

\footnotetext{
* e-mail: cuntz@uta.edu
}

the wave parameters, particularly the wave period, and that the shock strength and energy dissipation rate is largely affected by the spreading of the tubes as function of height. Further results for different types of stars have been given by Cuntz et al. $(1998,1999)$ and Fawzy et al. (2002a,b). For recent updates on the theory of chromospheric heating see reviews by Ulmschneider \& Musielak (2003) and Musielak (2004).

The similarities between acoustic waves, acoustic tube waves and longitudinal tube waves is a strong motivation to also explore the properties of those waves through analytic methods. This has been done extensively for acoustic waves and has been described in detail in many standard text books (Mihalas \& Mihalas 1984; Landau \& Lifshitz 1987, and references therein). Similar studies for longitudinal tube waves are given in this paper series. In Paper I, Cuntz (1999) presented analytic shock amplitude relations for various quantities such as pressure, density, temperature, velocity, magnetic field strength and the tube cross section. Due to the extreme coupling of the Rankine-Hugoniot relations, those relationships could not be separated with the shock strength as free parameter, contrary to the case of acoustic waves. In case of weak shocks, however, the Rankine-Hugoniot relationships could successfully be decoupled allowing detailed evaluations. Note that Paper I only deals with the amplitude relations across shocks with the preshock variables being specified, but not with properties related to the propagation of shocks.

An important property of acoustic waves in gravitational atmospheres of plane-parallel geometry is that fully developed 
sawtooth shocks grow to a limiting strength (Ulmschneider 1970; Cuntz \& Ulmschneider 1988). The limiting shock strength property can be utilized for estimating the heating potential of the waves at large heights. This approach has already been used, for instance, to assess the possibility of acoustic waves to heat stellar coronae (Stępień \& Ulmschneider 1989) and to drive winds in low-gravity stars (Cuntz 1990). To find out whether longitudinal tube waves also show limiting shock strength behavior is very relevant for checking the possible role of those waves for heating higher chromospheric regions as well as transition layers in different types of stars including the Sun. In the present work we derive limiting shock strength formulas for longitudinal tube waves and discuss the conditions of applicability. Section 2 outlines the magnetohydrodynamic equations. In Sect. 3, we derive the limiting shock strength formula for longitudinal tube waves, whereas in Sect. 4 we give comparisons with results from time-dependent wave computations. Conclusions are given in Sect. 5.

\section{Magnetohydrodynamic equations}

\subsection{Rankine-Hugoniot relations}

In accordance to Paper I, we again consider a thin, vertically directed magnetic flux tube embedded in a non-magnetic external medium. The Rankine-Hugoniot relations for longitudinal tube waves are given by

$\rho_{1} A_{1} v_{1}=\rho_{2} A_{2} v_{2}=j$,

$A_{1}\left(\rho_{1} v_{1}^{2}-2 p_{\mathrm{e}}+2 p_{1}\right)=A_{2}\left(\rho_{2} v_{2}^{2}-2 p_{\mathrm{e}}+2 p_{2}\right)$,

$\frac{1}{2} v_{1}^{2}+H_{1}=\frac{1}{2} v_{2}^{2}+H_{2}$

Here $\rho_{1}$ is the density, $A_{1}$ is the tube cross section, $v_{1}$ the gas velocity, $p_{1}$ the gas pressure and $H_{1}$ the enthalpy in front of the shock. Values with index 2 refer to the state behind the shock. $j$ is the mass flux and $p_{\mathrm{e}}$ the gas pressure of the field free region outside the tube. The velocities $v_{1}$ and $v_{2}$ are measured in the shock frame with the transformation of the gas velocity from the laboratory (Euler) frame into the shock frame given by

$v_{1}=u_{1}-U_{\mathrm{sh}}, \quad v_{2}=u_{2}-U_{\mathrm{sh}}$.

Here $U_{\text {sh }}$ is the shock speed in the laboratory frame. We also assume that the gas velocities $u_{1}, u_{2}$ are related by $u_{2}=-u_{1}$, implying that the mean velocity $u_{\mathrm{o}}$ at the shocks (Euler frame) is zero.

The shock strength $M_{\mathrm{s}}$ is given by

$M_{\mathrm{s}}=\frac{U_{\mathrm{sh}}-u_{1}}{c_{\mathrm{T} 1}}=1+\hat{\alpha}$,

where $u_{1}$ and $c_{\mathrm{T} 1}$ is the flow speed and tube speed ${ }^{1}$ in front of the shock, respectively, and $\hat{\alpha}$ is the residual shock strength for weak shocks. As noted in Paper I, this definition deviates slightly from the case of acoustic shocks, because here the Mach number is given in terms of $c_{\mathrm{T} 1}$ instead of $c_{\mathrm{S} 1}$.

\footnotetext{
1 This definition also corrects a typo following Eq. (15) of Paper I.
}

We introduce the normalized shock amplitudes $u_{\mathrm{m}}^{\prime}, \rho_{\mathrm{m}}^{\prime}, p_{\mathrm{m}}^{\prime}$, and $c_{\mathrm{Sm}}^{\prime}$ given by $u_{\mathrm{m}}^{\prime}=u_{\mathrm{m}} / c_{\mathrm{To}}, \rho_{\mathrm{m}}=\rho_{\mathrm{m}} / \rho_{\mathrm{o}}$, and so on. The quantities with subscript " $\mathrm{m}$ " are the amplitudes at the shock given by, e.g., $\rho_{\mathrm{m}}=\left(\rho_{2}-\rho_{1}\right) / 2$, whereas the quantities with subscript "o" represent the mean quantities at the shock, e.g., $\rho_{\mathrm{o}}=\left(\rho_{1}+\rho_{2}\right) / 2$. Following Paper I, we find

$$
\begin{aligned}
& u_{\mathrm{m}}^{\prime}=\frac{2}{\gamma+1} \xi \hat{\alpha} \\
& \rho_{\mathrm{m}}^{\prime}=\frac{2}{\gamma+1}\left(1-\frac{c_{\mathrm{To}}^{2}}{c_{\mathrm{Ao}}^{2}}\right) \xi \hat{\alpha} \\
& p_{\mathrm{m}}^{\prime}=\frac{2 \gamma}{\gamma+1}\left(1-\frac{c_{\mathrm{To}}^{2}}{c_{\mathrm{Ao}}^{2}}\right) \xi \hat{\alpha}
\end{aligned}
$$

among other relations. Here $c_{\mathrm{To}}$ and $c_{\mathrm{Ao}}$ denote the mean values of the tube speed and Alfvén speed across the shock, respectively. For the amplitude parameter $\xi$ we have

$\xi=\frac{(\gamma+1)\left(1+\frac{1}{2} \beta \gamma\right)^{2}}{1+\frac{3}{2} \beta \gamma+\gamma}$,

which solely depends on plasma- $\beta$ (see below). Note that the ratio $c_{\mathrm{To}} / c_{\mathrm{Ao}}$ can also be expressed in terms of $c_{\mathrm{To}}$ and $c_{\mathrm{So}}$ or, alternatively, by plasma- $\beta$ as

$\frac{c_{\mathrm{To}}^{2}}{c_{\mathrm{Ao}}^{2}}=1-\left(1+\frac{1}{2} \beta \gamma\right)^{-1}$

(see Eq. (41); Paper I) with $c_{\mathrm{S}}$ as adiabatic sound speed and $c_{\mathrm{A}}$ as Alfvén speed.

For general values of $\rho, p, c_{\mathrm{A}}, c_{\mathrm{S}}$, and $B$, various relationships exist. The Alfvén speed $c_{\mathrm{A}}$ is given by

$c_{\mathrm{A}}=\frac{B}{\sqrt{4 \pi \rho}}$.

By introducing plasma- $\beta$, we find

$\beta=\frac{p}{p_{\mathrm{e}}-p}=\frac{2 p}{\rho c_{\mathrm{A}}^{2}}$

The enthalpy $H$ is given by

$H=\frac{\gamma}{\gamma-1} \frac{p}{\rho}=\frac{c_{\mathrm{S}}^{2}}{\gamma-1}$.

In Eq. (13), we considered the ideal gas law, as we assume a neutral gas with a mean molecular weight of $\mu=1.3 \mathrm{~g} \mathrm{~mol}^{-1}$ and $\gamma=5 / 3$ as ratio of the specific heats. Note that $c_{\mathrm{S}}$ is related to $c_{\mathrm{A}}$ and the tube speed $c_{\mathrm{T}}$ by

$\frac{1}{c_{\mathrm{T}}^{2}}=\frac{1}{c_{\mathrm{S}}^{2}}+\frac{1}{c_{\mathrm{A}}^{2}}$.

With Eq. (12) we also obtain

$c_{\mathrm{S}}=c_{\mathrm{T}} \sqrt{1+\frac{1}{2} \beta \gamma}$.

The comparison between $c_{\mathrm{S}}$ and $c_{\mathrm{T}}$ is given in Fig. 1 . 


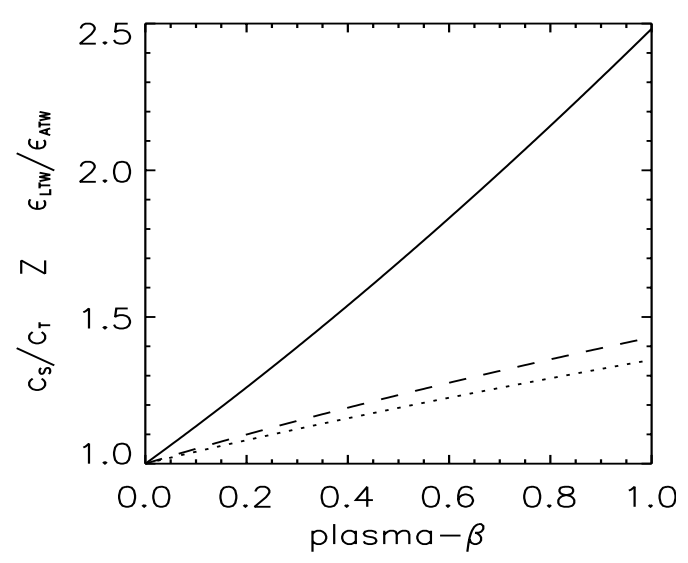

Fig. 1. Behavior of $c_{\mathrm{S}} / c_{\mathrm{T}}$ (dotted), $\mathcal{Z}$ (dashed), and $\epsilon_{\mathrm{LTW}} / \epsilon_{\mathrm{ATW}}$ (solid) as function of plasma- $\beta$. The corresponding equations are (15), (38), and (40).

\subsection{Energy dissipation rate and wave energy flux}

We also need to evaluate the energy dissipation rate $\epsilon$. Following Herbold et al. (1985), we find

$\epsilon=\frac{\rho_{\mathrm{o}} T_{\mathrm{o}} \Delta S}{P}$.

Here $\Delta S$ is the entropy jump at the shock, $P$ the wave period, and $\rho_{\mathrm{o}}$ and $T_{\mathrm{o}}$ the mean density and temperature at the shock, respectively. We also find

$\Delta S=\left.\frac{1}{12 T_{1}}\left(\frac{\partial^{2}(1 / \rho)}{\partial p^{2}}\right)\right|_{1}\left(p_{2}-p_{1}\right)^{3}$

leading to

$\Delta S=\frac{1}{12} \frac{\gamma+1}{\gamma^{2}} \frac{\Re}{\mu} \frac{\left(p_{2}-p_{1}\right)^{3}}{p_{1}^{3}}$

(e.g., Landau \& Lifshitz 1987) with all symbols having their usual meaning. Here we assumed that $A_{1} \simeq A_{2}$. Equation (18) must now be expanded for weak shocks. For $p_{2} / p_{1}$ we make use of Eq. (8) noting that $p_{2} / p_{1}=2 p_{\mathrm{m}}^{\prime}$ in lowest order. The dissipation rate $\epsilon$ is then given by

$\epsilon=\frac{16}{3} \frac{\gamma(\gamma+1)\left(1+\frac{1}{2} \beta \gamma\right)^{3} p_{\mathrm{o}}}{\left(1+\frac{3}{2} \beta \gamma+\gamma\right)^{3} P} \hat{\alpha}^{3}$

with $p_{\mathrm{o}}$ as mean pressure of the atmosphere. (Note that in case of small amplitude shock waves, the mean quantities at the shock and the mean quantities of the atmosphere coincide.) Obviously, the dissipation rate for longitudinal flux tube waves depends on the third power of $\hat{\alpha}$, which is equivalent to the case of acoustic waves (see Appendix A).

Figure 2 depicts the dissipation rate $\Delta S$ (Eq. (18)) as function of the shock strength. Here we show the results from the exact solution of the Rankine-Hugoniot equations (see Eqs. (1)-(3); i.e., not assuming the weak shock expansion for the shock amplitudes) in comparison with the approximation given by Eq. (19) for the case $\beta=0$ (acoustic case) and $\beta=0.5$. Very surprisingly, it is found that the approximate value of $\Delta S$ for $\beta=0$ is larger than the approximate value for $\beta=0.5$ even though the exact value of $\Delta S$ for $\beta=0$ is always lower than

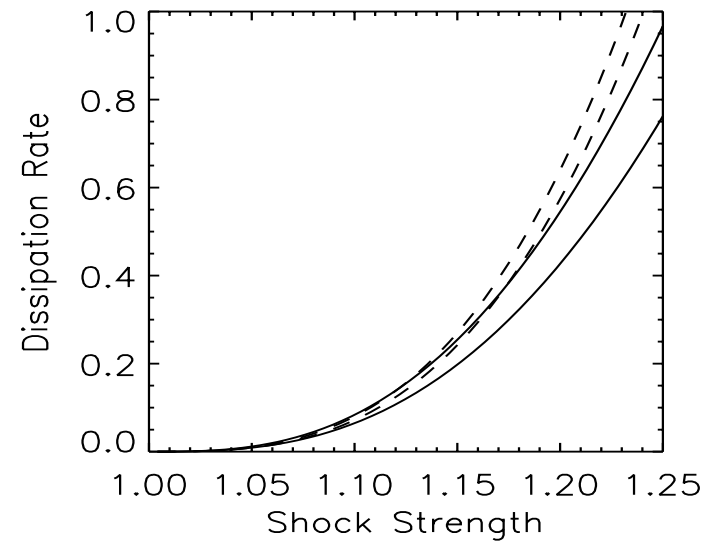

Fig. 2. Dissipation rate $\Delta S\left(\times 10^{6} \mathrm{erg} \mathrm{g}^{-1} \mathrm{~K}^{-1}\right)$ for shocks of longitudinal flux tube waves given by the Rankine-Hugoniot relations as function of shock strength $M_{s}$. The exact values are given as solid lines for $\beta=0$ (upper line) and $\beta=0.5$ (lower line), whereas the approximations following Eq. (18) are given as dashed lines for $\beta=0$ (lower line) and $\beta=0.5$ (upper line).

the exact value attained for $\beta=0.5$. This means, on one hand, that the approximation improves for higher values of plasma- $\beta$ and that it is always better than the well-established approximation in case of plasma- $\beta=0$, i.e., the acoustic case (see Appendix A). On the other hand, the differential dependence of $\Delta S$ on $\beta$ is obviously incorrectly represented. Therefore, we will ignore terms on the order of $\mathrm{d} \beta / \mathrm{d} r$ in the subsequent expansion of the limiting shock strength (see Sect. 3), as otherwise we would obtain a limiting shock strength formula of lesser quality.

Next, we derive the expression of the wave energy flux for a sawtooth-type shock wave in a gravitational atmosphere. The wave energy flux is given as

$\mathcal{F}_{\mathrm{M}}{ }^{\text {wave }}=\rho u A\left(\frac{1}{2} u^{2}+H+\phi\right)$

consisting of the kinetic energy flux, enthalpy flux and potential energy flux. We neglect the first term in the parenthesis because it is a third-order term. Next, we need the mean value

$\mathcal{F}_{\mathrm{M}}=\overline{\rho u A H}+\overline{\rho u A} \phi$.

Assuming that $A=A_{\mathrm{o}}, H=H_{\mathrm{o}}+\delta H$, and noting that $\delta H=$ $(\partial H / \partial p)_{\mathrm{s}} \delta p=\delta p / \rho$, we get

$\mathcal{F}_{\mathrm{M}}=\overline{\rho u A} H_{\mathrm{o}}+\overline{u A \delta p}+\overline{\rho u A} \phi$.

We further assume that the average mass flux $\overline{\rho u A}$ is identical zero. We thus obtain

$\mathcal{F}_{\mathrm{M}}=\overline{u A \delta p}$

and

$\overline{u A \delta p}=\frac{A}{P} \int_{0}^{P} \delta p u \mathrm{~d} t$

with $P$ as wave period and $\delta p=p-p_{\mathrm{o}}$.

Considering that the wave energy flux per unit area is given by $F_{\mathrm{M}}=\mathcal{F}_{\mathrm{M}} / A$, we find

$F_{\mathrm{M}}=\frac{1}{P} \int_{0}^{P}\left(p-p_{\mathrm{o}}\right) u \mathrm{~d} t$. 
As we consider sawtooth shock waves, we write $p=p_{\mathrm{o}}-p_{\mathrm{m}}+$ $2 p_{\mathrm{m}} t / P$ and $u=-u_{\mathrm{m}}+2 u_{\mathrm{m}} t / P$; therefore, $F_{\mathrm{M}}$ reads

$F_{\mathrm{M}}=\frac{p_{\mathrm{m}} u_{\mathrm{m}}}{P} \int_{0}^{P}\left(1-\frac{2 t}{P}\right)^{2} \mathrm{~d} t=\frac{1}{3} p_{\mathrm{m}} u_{\mathrm{m}}$.

Substituting $p_{\mathrm{m}}, u_{\mathrm{m}}$ by Eqs. (8) and (6), we obtain with $p_{\mathrm{m}}=$ $p_{\mathrm{m}}^{\prime} p_{\mathrm{o}}$ and $u_{\mathrm{m}}=u_{\mathrm{m}}^{\prime} c_{\mathrm{To}}$ and $\xi$ given by Eq. (9)

$F_{\mathrm{M}}=\frac{4 \gamma\left(1+\frac{1}{2} \beta \gamma\right)^{3} p_{\mathrm{o}} c_{\mathrm{To}}}{3\left(1+\frac{3}{2} \beta \gamma+\gamma\right)^{2}} \hat{\alpha}^{2}$.

Alternatively, we also obtain

$F_{\mathrm{M}}=\frac{1}{12} \gamma\left(1+\frac{1}{2} \beta \gamma\right) p_{\mathrm{o}} c_{\mathrm{To}} \hat{\eta}^{2}$

from the relation between $\hat{\eta}$ and $\hat{\alpha}$ (see Eq. (48); Paper I) with $\hat{\eta}$ given by $\hat{\eta}=\left(\rho_{2}-\rho_{1}\right) / \rho_{1}$.

\section{Limiting shock strength}

To derive the limiting shock strength $\hat{\alpha}_{\text {lim }}$, we start from the continuity equation for generalized geometry with dissipation (e.g., Mihalas \& Mihalas 1984; Pozrikidis 1997) applied to the wave energy flux

$\frac{\mathrm{d} F_{\mathrm{M}}}{\mathrm{d} z}+\frac{F_{\mathrm{M}}}{A} \frac{\mathrm{d} A}{\mathrm{~d} z}=-\epsilon$

Note that plasma- $\beta$ may change with height depending on the type of flux tube. In this case, the relation between $\mathrm{d} c_{\mathrm{To}} / \mathrm{d} z$ and $\mathrm{d} c_{\text {So }} / \mathrm{d} z$ follows from Eqs. (14), (15) as

$\frac{1}{c_{\mathrm{To}}} \frac{\mathrm{d} c_{\mathrm{To}}}{\mathrm{d} z}=\frac{1}{c_{\mathrm{So}}} \frac{\mathrm{d} c_{\mathrm{So}}}{\mathrm{d} z}-\frac{1}{2}\left(1+\frac{1}{2} \beta \gamma\right)^{-1} \frac{\mathrm{d} \beta}{\mathrm{d} z}$.

With Eqs. (27), (29), and (30), we find

$\frac{\mathrm{d} F_{\mathrm{M}}}{\mathrm{d} z}=F_{\mathrm{M}}\left(\frac{2}{\hat{\alpha}} \frac{\mathrm{d} \hat{\alpha}}{\mathrm{d} z}+\frac{3}{2} \frac{1}{H_{\mathrm{T}}}-\frac{1}{H_{\rho}}\right)$

with $H_{\mathrm{T}}$ and $H_{\rho}$ as scale height for the temperature and density, respectively, given by

$H_{\mathrm{T}}=\left|\frac{1}{T_{\mathrm{o}}} \frac{\mathrm{d} T_{\mathrm{o}}}{\mathrm{d} z}\right|^{-1}$

and

$H_{\rho}=\left|\frac{1}{\rho_{\mathrm{o}}} \frac{\mathrm{d} \rho_{\mathrm{o}}}{\mathrm{d} z}\right|^{-1}=\frac{c_{\mathrm{So}}^{2}}{\gamma g}$.

The scale height for plasma- $\beta$ is given by

$H_{\beta}=\left|\frac{1}{\beta} \frac{\mathrm{d} \beta}{\mathrm{d} z}\right|^{-1}$.

As we are mostly concerned with the treatment of longitudinal flux tube waves in stellar chromospheres, we assume that the mean temperature $T_{\mathrm{o}}$ increases with height, whereas the mean density $\rho_{\mathrm{o}}$ and plasma- $\beta$ decrease with height. Therefore, we must use $+H_{\mathrm{T}}^{-1}$ and $-H_{\rho}^{-1}$ in Eq. (31). For other applications, the signs need to be adjusted accordingly. Usually, we also have $H_{\mathrm{T}} \gg H_{\rho}$.
With Eqs. (19), (27), (29), and (31), we can deduce the ODE for $\hat{\alpha}$ given by

$\frac{2}{\hat{\alpha}} \frac{\mathrm{d} \hat{\alpha}}{\mathrm{d} z}+\frac{3}{2} \frac{1}{H_{\mathrm{T}}}-\frac{1}{H_{\rho}}+\frac{1}{A} \frac{\mathrm{d} A}{\mathrm{~d} z}=-\frac{4(\gamma+1) \hat{\alpha}}{\left(1+\frac{3}{2} \beta \gamma+\gamma\right) c_{\mathrm{To}} P}$

with terms on the order of $H_{\beta}^{-1}$ omitted (see Sect. 2).

In case of plane-parallel geometry, i.e., constant crosssection tubes, we have $1 / A \mathrm{~d} A / \mathrm{d} z=0$. For exponential tubes with $A=A_{\mathrm{oo}} \exp (z / L)$, we have $1 / A \mathrm{~d} A / \mathrm{d} z=L^{-1}$. Here $L$ denotes the scale height of the geometrical dilution given by

$L=\left|\frac{1}{A} \frac{\mathrm{d} A}{\mathrm{~d} z}\right|^{-1}$.

Note that with the exception of constant cross-section tubes and exponential tubes, $L$ still depends on $z$. In this case, the solution for $\hat{\alpha}(z)$ and $\hat{\alpha}_{\text {lim }}$, i.e., the asymptotic behavior of $\hat{\alpha}$ for large $z$, requires to solve a Riccati ODE of non-constant coefficients (see Appendix B). Nonetheless, it is still possible to deduce an approximate expression for $\hat{\alpha}_{\mathrm{lim}}$. If the dependence of $L$ on $z$ is weak, we find $\mathrm{d} \hat{\alpha} / \mathrm{d} z \simeq 0$ and

$\hat{\alpha}_{\mathrm{lim}} \simeq \frac{c_{\mathrm{So}} P \mathcal{Z}}{4}\left(\frac{1}{H_{\rho}}-\frac{3}{2} \frac{1}{H_{\mathrm{T}}}-\frac{1}{L}\right)$

for large values of $z$.

In Eq. (36), we chose $L^{-1}$ instead of $-L^{-1}$ as we assumed that the flux tube area $A(z)$ increases as function of height. For simplicity, we also introduced the function $\mathcal{Z}(\beta)$ given by

$\mathcal{Z}(\beta)=\frac{1+\frac{3}{2} \beta \gamma+\gamma}{(\gamma+1)\left(1+\frac{1}{2} \beta \gamma\right)^{\frac{1}{2}}}$,

which solely depends on plasma- $\beta$ (see Fig. 1). An expression equivalent to Eq. (37) can be obtained for the limiting value of the density jump $\hat{\eta}_{\text {lim }}$ using again the relation between $\hat{\eta}$ and $\hat{\alpha}$ as derived in Paper I. Note that for constant cross-section tubes and exponential tubes Eq. (37) is exact. The function $\mathcal{Z}(\beta)$ indicates perfect equivalence between longitudinal tube waves and acoustic tube waves. For $\beta \rightarrow 0$, we find $\mathcal{Z} \rightarrow 1$, which corresponds to the case of a flux tube being almost completely evacuated. In this case, the behavior of a longitudinal tube wave is essentially that of an acoustic wave.

A further expression to be evaluated is the scale height $H_{F}$ of the decrease of the waves energy flux $F_{\mathrm{M}}$ due to energy dissipation, geometrical dilution, or both (see Eq. (31)). With $\mathrm{d} F_{\mathrm{M}} / \mathrm{d} z=-F_{\mathrm{M}} / H_{F}$ we find

$\frac{1}{H_{F}}=\frac{1}{H_{\rho}}-\frac{3}{2} \frac{1}{H_{\mathrm{T}}}-\frac{1}{L}$.

In addition, we can also calculate the ratio between the energy dissipation rate for longitudinal flux tube waves and acoustic tube waves of limiting shock strength. In this case, we need to compare the energy dissipation rate given by Eqs. (19) and (A.3). For tubes of identical geometry, the term given by the geometrical dilution is identical irrespective of the type of wave. With Eqs. (37), (38), and (A.5), we find

$\frac{\epsilon_{\mathrm{LTW}}}{\epsilon_{\mathrm{ATW}}}=\frac{c_{\mathrm{To}}^{-3}}{c_{\mathrm{So}}^{-3}}=\left(1+\frac{1}{2} \beta \gamma\right)^{\frac{3}{2}}$. 


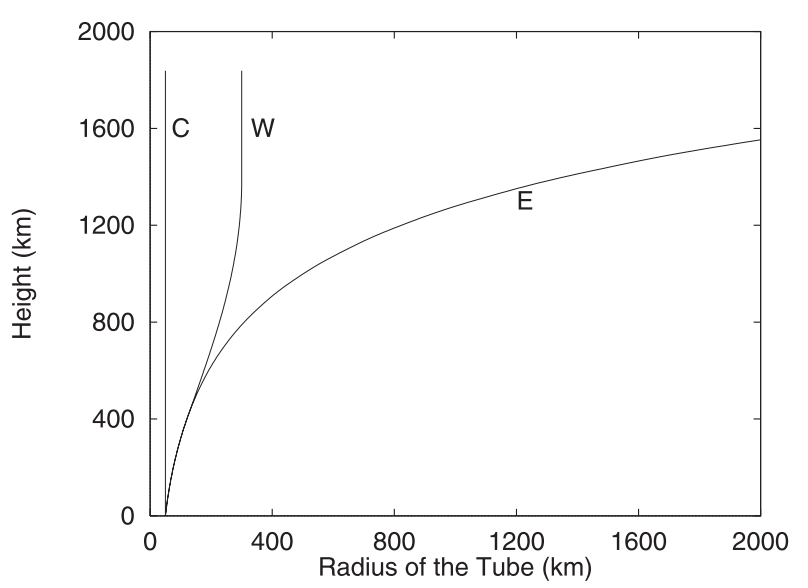

Fig. 3. Shape of the constant cross-section (C), wine-glass (W), and exponential flux tube (E) used in the present work. The radii of the tubes are shown as function of height (adopted from Fawzy et al. 1998).

Obviously, the ratio between the energy dissipation rates of longitudinal and acoustic tube waves solely depends on plasma- $\beta$ (see Fig. 2), indicating a higher dissipation rate for longitudinal tube waves. The limiting shock strength of longitudinal tube waves $1+\hat{\alpha}_{\text {lim }}$ is given by Eq. (37), whereas the limiting shock strength for acoustic tube waves $1+\alpha_{\text {lim }}$ follows from Eq. (A.5). It is found that the shock strength of longitudinal tube waves is always higher than that of acoustic tube waves implying that those waves have a greater heating potential.

\section{Comparison with time-dependent wave computations}

\subsection{Flux tube models}

In our study we consider three different magnetic flux tube models, which are: a constant cross-section, wine-glass, and an exponential tube (see Fig. 3). These tubes largely differ in shape and have been chosen to investigate the influence of different geometrical dilution factors on the limiting shock strength behavior of the waves. These flux tube models have already been used by Fawzy et al. (1998) to study the propagation of longitudinal flux tube waves in the solar chromosphere. The tubes are embedded in a non-grey radiative and hydrostatic equilibrium atmosphere. Initially, there is no mechanical heating. At height $z=0$, where in the external atmosphere the optical depth is $\tau_{5000}=1$, the tubes have a specified radius $r_{\mathrm{B}}=50 \mathrm{~km}$ and a magnetic field strength $B_{\mathrm{oo}}=1500 \mathrm{G}$. The exponential tube has exponential spreading up to the top, whereas the wine-glass tube has exponential spreading up to $400 \mathrm{~km}$, smaller spreading at intermediate heights, and no spreading beyond $1400 \mathrm{~km}$.

Note that the top tube opening radii $r_{\mathrm{T}}$ are chosen differently for the different types of tubes, whereas the bottom opening radii $r_{\mathrm{B}}$ are identical. For the constant cross-section tube, we take $r_{\mathrm{T}}=r_{\mathrm{B}}$. The two other tubes are allowed to spread with height in pressure balance with the surrounding medium. To ensure that the wine-glass tube has its desired radius, an additional time-independent external pressure is implied beyond $400 \mathrm{~km}$, which is assumed to originate from magnetic interaction with neighboring tubes. The exponential tube, characterized by very extreme spreading, has a height of $1580 \mathrm{~km}$ and a tube opening radius of $2155 \mathrm{~km}$. The wine-glass tube has a height of $1820 \mathrm{~km}$ and a tube opening radius of $300 \mathrm{~km}$. Although not entirely realistic, the exponential tube is important for testing our limiting shock strength formula in case of exponential spreading. The exponential tube with its unobstructed spreading may be typical for tubes in the interior of supergranulation cells and in areas just outside the network region, whereas the constant cross-section tube may represent the inner part of a highly overcrowded network region. Wine-glass tubes, on the other hand, with a filling factor of $f=r_{\mathrm{B}}^{2} / r_{\mathrm{T}}^{2} \approx 2.8 \%$ correspond to flux tubes observed in the outer parts of a typical network region (see Fawzy et al. 1998).

Figure 4 (middle and lower panel) shows the magnetic field strength and plasma- $\beta$ as function of height in the undisturbed tube models. The behavior of the density is identical in all three tube models with $H_{\rho} \simeq 110 \mathrm{~km}$ as density scale height. The temperature scale heights $H_{\mathrm{T}}$ in the models exceed $H_{\rho}$ by more than a factor of 30 . The change of the undisturbed magnetic field strength $B_{0}(z)$ with height is dictated by the area function $A_{\mathrm{o}}(z)$ as the result of the magnetic flux conservation $B_{\mathrm{o}} A_{\mathrm{o}}=\phi$ (see Eq. (17), Paper I). Plasma- $\beta$ is constant with height in case of the exponential tube, but decreases exponentially in the constant cross-section tube with a scale-height of $H_{\beta} \simeq 110 \mathrm{~km}$. The behavior of the magnetic field strength and of plasma- $\beta$ in the wine-glass tube is intermediate between the constant cross-section and the exponential tube, as expected.

\subsection{Assessment of the limiting shock strengths}

Figure 4 (upper panel) depicts the behavior of the limiting shock strength $M_{\mathrm{s}}^{\mathrm{lim}}=1+\hat{\alpha}_{\mathrm{lim}}$ as function of height (see Eq. (37)). Here we assumed a wave period of $P=30 \mathrm{~s}$. The adiabatic sound speed $c_{S \text { o }}$ used for the computation ranges between $6.7 \times 10^{5}$ and $8.0 \times 10^{5} \mathrm{~cm} \mathrm{~s}^{-1}$, depending on the height. Determining the limiting shock strength requires the computation of $H_{\rho}, H_{\mathrm{T}}$, and $L$, i.e., the scale-heights for the density, temperature, and flux tube area, respectively (see Eqs. (33), (32), (36)). As we have $H_{\mathrm{T}} \gg H_{\rho}$, we can assume $H_{\mathrm{T}}^{-1}=0$ and $H_{\rho} \simeq H_{\mathrm{p}}$ with $H_{\mathrm{p}}$ as pressure scale height. The waviness of the shock strengths (see Fig. 4) is due to remaining inaccuracies in the temperature and flux correction methods employed in the calculation of the initial radiative equilibrium atmosphere models (Cuntz et al. 1994).

Now we compare the analytically deduced limiting shock strengths for the different types of tubes. It is found that for the constant cross-section tube and the exponential tube $M_{\mathrm{s}}^{\mathrm{lim}}$ does not change with height. The deduced values of $M_{\mathrm{s}}^{\mathrm{lim}}$ are 1.34 and 1.51 , respectively. In case of the wine-glass tube, however, no height-independent value for $M_{\mathrm{s}}^{\mathrm{lim}}$ is found in the region of tube spreading. In this type of tube, $M_{\mathrm{s}}^{\mathrm{lim}}$ approaches the value for the exponential tube at low heights and that for the constant cross-section tube at large heights. This behavior is expected as for the wine-glass tube $M_{\mathrm{S}}$ is close to that of the exponential tube at low heights, where it is exponentially spreading, and 

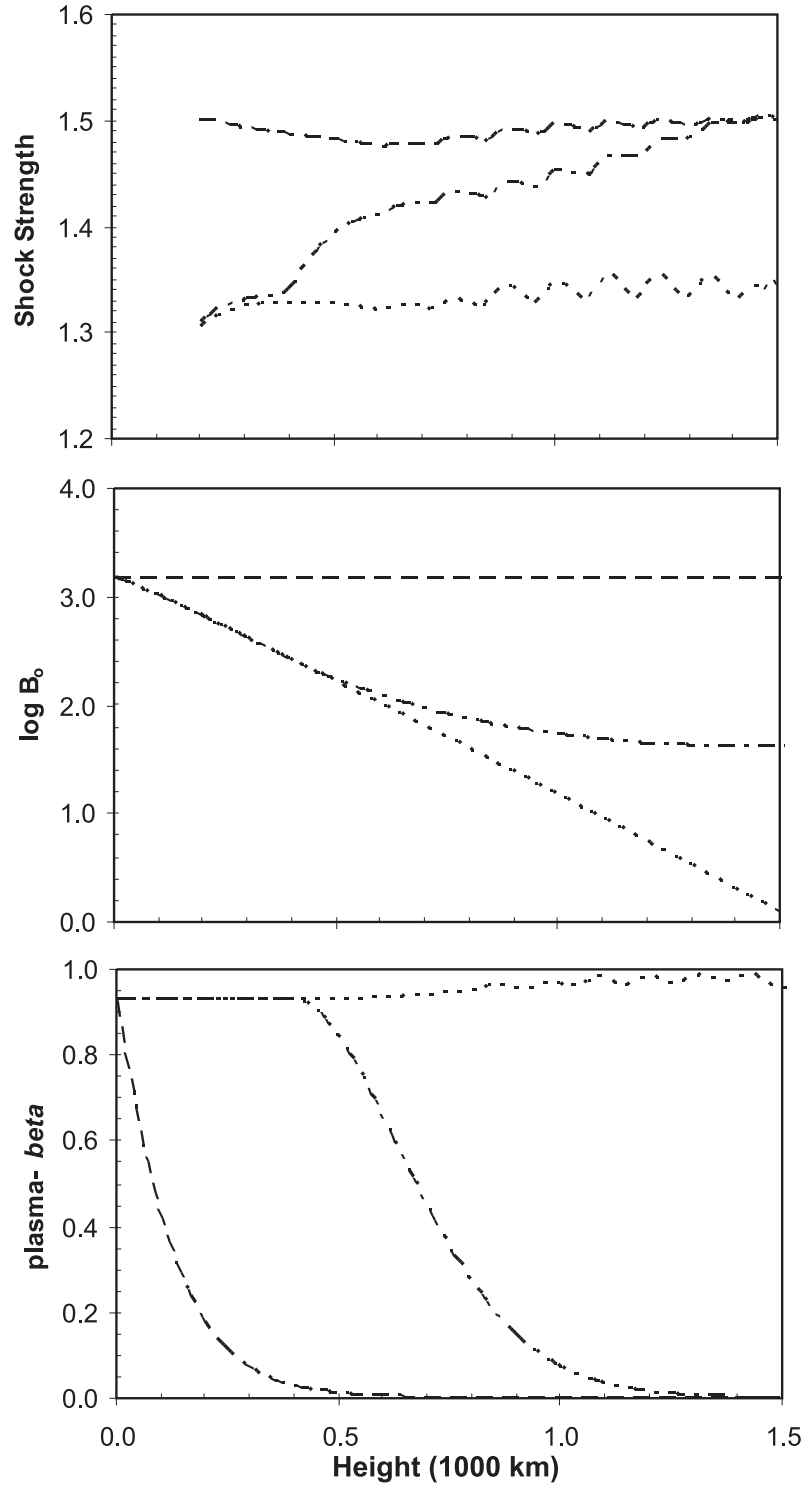

Fig. 4. Behavior of the limiting shock strength $M_{s}^{\text {lim }}$, the magnetic field strength $B_{\mathrm{o}}$ and plasma- $\beta$ in the undisturbed tube models (see text). Shown are the constant cross-section tube (dashed), wine-glass tube (dashed-dotted), and exponential tube (dotted). The density structure is identical in all three models.

close to that of the constant cross-section tube at large height, where the cross section stays constant (see Fig. 3).

The fact that $M_{\mathrm{s}}^{\mathrm{lim}}$ is independent of height in case of the constant cross-section tube and exponential tube can easily be understood by inspecting Eq. (37). In all models, the density scale height is constant with $H_{\rho} \simeq 110 \mathrm{~km}$ (see Sect. 4.1). In case of the constant cross-section tube, we have $L^{-1}=0$ and incidently we also have $H_{\rho} \simeq H_{\beta}$. As a consequence, we find that the RHS of Eq. (37) is constant and thus independent of height. For the exponential tube, we have $H_{\rho} \simeq L / 2$, and therefore we find again that $H_{\rho}$ and $L$ are independent of height. Consequently, the RHS of Eq. (37) is again identified to be independent of height, resulting in a constant value for $M_{\mathrm{s}}^{\mathrm{lim}}$. On the other hand, for the wine-glass tube, $L$ changes with height in some parts of the atmosphere due to the behavior of the area function $A(r)$ leading to a height-dependent limiting shock strength.

\subsection{Analytical results versus simulations}

The analytic predictions of the limiting shock strength $M_{\mathrm{s}}^{\lim }$ can be compared with results from time-dependent simulations. Therefore, we use a time-dependent MHD computer code previously described by Rammacher (1991), Cuntz et al. (1999), and Fawzy et al. (2002a). Longitudinal tube waves are introduced at the bottom of the flux tubes, and are followed to the point of shock formation and beyond. In the following, we assume initial wave energy fluxes of $F_{\mathrm{M}}=10^{6}, 10^{7}$, and $10^{8} \mathrm{erg} \mathrm{cm}^{-2} \mathrm{~s}^{-1}$ and a wave period of $P=30 \mathrm{~s}$ as in the analytic models.

The time-dependent wave models show the following behavior: in case of the constant cross section tube, it is found that shocks are formed at 790,560 , and $330 \mathrm{~km}$ for waves with an initial energy of $10^{6}, 10^{7}$, and $10^{8} \mathrm{erg} \mathrm{cm}^{-2} \mathrm{~s}^{-1}$, respectively, confirming that a higher wave energy leads to shock formation at a lower atmospheric height. For the exponential tube, the shock formation heights are 1350,730, and $340 \mathrm{~km}$, respectively. The wine-glass tube constitutes an intermediate case, as expected. All these results are fully explanable by the geometrical dilution of the wave energy flux in the different types of tubes, which both affects time-dependent and analytical longitudinal wave models. The shock strengths representing the time-dependent wave models (see Fig. 5) were obtained after 30 to 60 wave periods had been inserted into the atmosphere, with the exact number of periods depending on the tube model as well as the initial wave energy flux.

Most importantly, our models allow to check whether the analytically deduced limiting shock strengths given as $M_{\mathrm{s}}^{\mathrm{lim}}=$ $1+\alpha_{\text {lim }}$ (see Eq. (37)) are consistent with those from timedependent simulations. We find that this is indeed the case (see Fig. 5). For the constant cross-section tube, $M_{\mathrm{s}}^{\mathrm{lim}}$ is attained at heights between 580 and $1350 \mathrm{~km}$ for wave energy fluxes be-

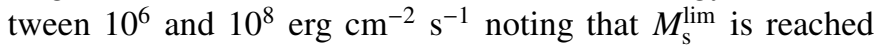
at low heights for high energy waves (and vice versa) as also found for acoustic waves (Cuntz \& Ulmschneider 1988). In case of the wine-glass tube, $M_{\mathrm{s}}^{\mathrm{lim}}$ is also reached at large heights as implied by the height evolution of the time-dependent shock strength. On the other hand, for the exponential tube, the analytically deduced limiting shock strength is not yet reached close to the upper end of the atmospheric domain at $1500 \mathrm{~km}$, even for waves with an initial energy flux of $10^{8} \mathrm{erg} \mathrm{cm}^{-2} \mathrm{~s}^{-1}$.

The reason for this behavior is that in case of exponential tubes an excessively large atmospheric volume must be heated by the waves (especially if compared to the other types of tubes), and that due to the relatively small tube bottom radius $r_{\mathrm{B}}=50 \mathrm{~km}$ (see Sect. 4.1), the area integrated wave energy flux $\mathcal{F}_{\mathrm{M}}=F_{\mathrm{M}} \cdot A$ is very low particularly if compared to the other tube models. To counteract this obstacle, we performed a test calculation with a very high flux of $F_{\mathrm{M}}=10^{9} \mathrm{erg} \mathrm{cm}^{-2} \mathrm{~s}^{-1}$ (see Fig. 5, bottom panel). In this case, the shock strengths given by the time-dependent simulations clearly approach the analytically deduced value of $M_{\mathrm{s}}^{\mathrm{lim}}$ near the upper end of the 

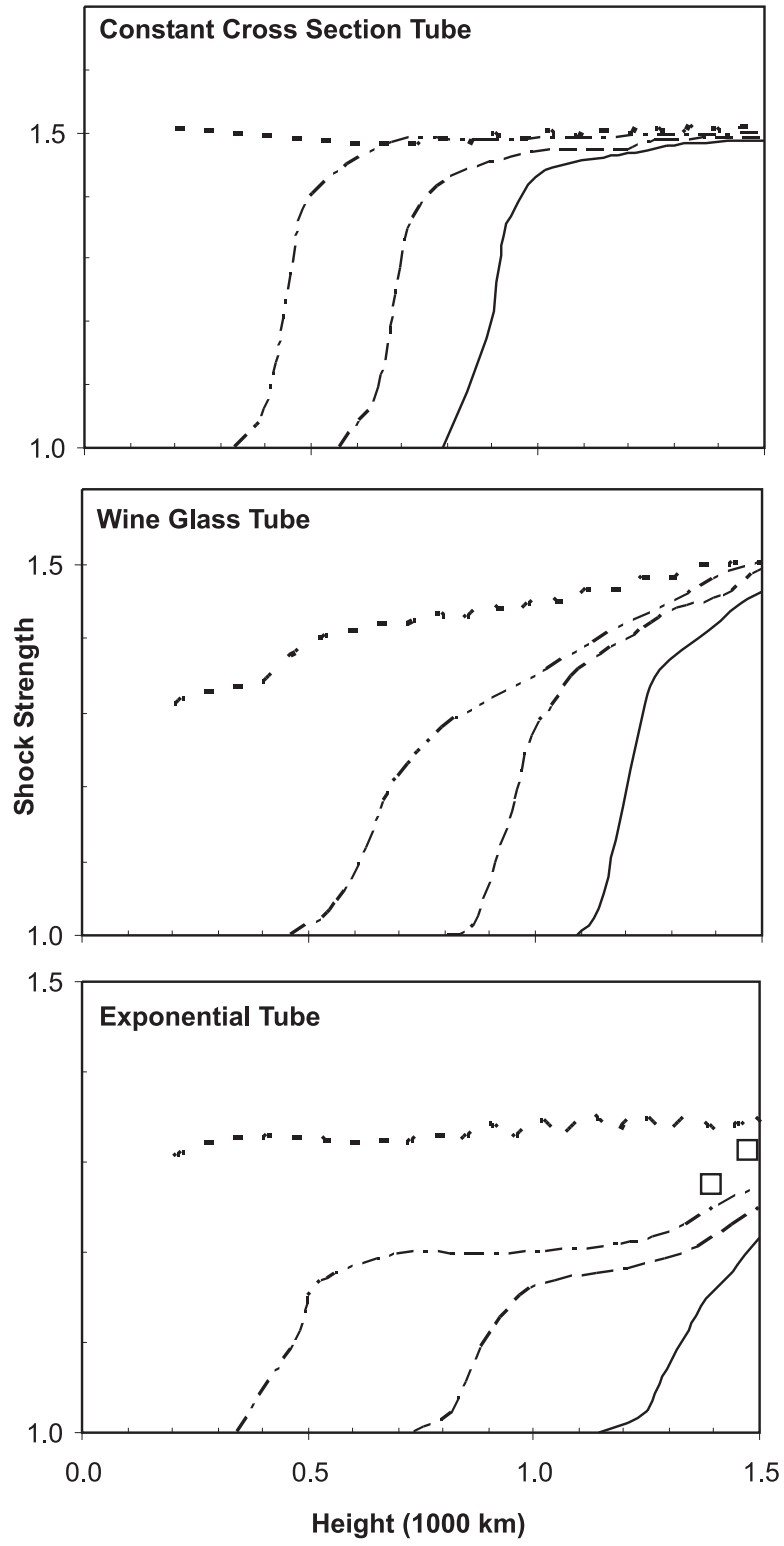

Fig. 5. Comparison of the analytically predicted limiting shock strength of longitudinal tube waves (see Eq. (37); bold-faced dashed) with results from time-dependent simulations for different initial wave energy fluxes. The initial wave energy fluxes are $F_{\mathrm{M}}=10^{6}$ (solid), $10^{7}$ (dashed), and $10^{8} \mathrm{erg} \mathrm{cm}^{-2} \mathrm{~s}^{-1}$ (dashed dotted). In case of the exponential tube, values for a wave energy flux of $F_{\mathrm{M}}=10^{9} \mathrm{erg} \mathrm{cm}^{-2} \mathrm{~s}^{-1}$ have been included (squares) for comparison. The adopted wave period is $P=30 \mathrm{~s}$ for all models.

atmospheric domain and agreement is expected to occur at heights between 1500 and $1800 \mathrm{~km}$.

\section{Conclusions}

In this paper, we derived an analytic expression for the limiting shock strength of longitudinal tube waves propagating in gravitational atmospheres assuming generalized flux tube geometry. Here we focused on constant cross-section tubes, wineglass tubes, and exponential tubes which were already used in previous studies (Fawzy et al. 1998). We also compared our analytic results with results from time-dependent numerical simulations. We found the following behavior:

1. A height-independent value for the limiting shock strength is found for constant cross-section tubes and exponential tubes. For wine-glass tubes, however, no heightindependent value for the limiting shock strength occurs in the region of tube spreading. In this type of tube, $M_{s}^{\lim }$ approaches the value for the exponential tube at low heights and that for the constant cross-section tube at large heights. These results are in excellent agreement with the findings by Fawzy et al. (1998).

2. The fact that the limiting shock strength does not change with height in case of constant cross-section tubes and exponential tubes follows directly from Eq. (37), which shows that the limiting shocks strengths depends solely on the scale heights of the density, temperature, and geometrical dilution and on plasma- $\beta$.

3. The derived limiting shock strength as well as the energy dissipation rate of longitudinal flux-tube waves show significant similarities to acoustic waves, particularly acoustic tube waves. This is due to the fact that acoustic tube waves can be identified with longitudinal tube waves through the limits plasma- $\beta \rightarrow 0$ and $\mathcal{Z} \rightarrow 1$ (see Paper I).

4. We derived a simple relationship between the energy dissipation rate of acoustic waves and longtitudinal waves. This ratio is found to be independent of the flux tube geometry and solely depends on plasma- $\beta$. For a given tube geometry, the dissipation rate of longitudinal waves is always higher.

5. The limiting strength was successfully reproduced in timedependent simulations for all three tube models. Timedependent models with relatively high initial wave energy fluxes ensured that the limiting shock strength was reproduced at a relatively low atmospheric height. In case of exponential tubes, a very high initial wave energy flux is needed to reproduce the limiting shock strength, owing to the fact that a very large atmospheric volume must be heated in comparison to the tube bottom area.

The results found in this paper allow to gauge the heating potential of longtitudinal tube waves without the necessity of detailed time-dependent simulations - an efforts already proved useful in studies of acoustic waves (e.g., Stępień \& Ulmschneider 1989). On the other hand, the derived results are strictly valid only for monochromatic waves of small amplitudes. The dynamics exhibited by wave frequency spectra (e.g., Musielak et al. 1995, 2000; Ulmschneider \& Musielak 1998; Ulmschneider et al. 2001) inevitably require detailed numerical modelling. Numerical simulations are also necessary in cases of large-amplitude waves (i.e., large shock strength) or long-period waves due to nonlinear effects.

Acknowledgements. The author acknowledges comments by $\mathrm{P}$. Ulmschneider, Z. E. Musielak, P. Rossi, and S. T. Suess as well as student support by T. Ahmed. This work has been supported by the UAH/USRA/NASA Cooperative Agreement on Research in Space Plasma Physics and by NSF under grant ATM-0087184. 


\section{Appendix A: Comparison with acoustic waves}

It is interesting to compare the limiting shock strength for longitudinal flux tube waves (see Sect. 3) with the result for acous$\mathrm{tic}^{2}$ waves. The wave energy flux for acoustic waves is given by

$F_{\mathrm{M}}=\frac{1}{12} \gamma p_{\mathrm{o}} c_{\mathrm{So}} \eta^{2}$

with

$\eta=\frac{4 \alpha}{\gamma+1}$

(Ulmschneider 1970). The energy dissipation rate $\epsilon$ is given by

$\epsilon=\frac{16}{3} \frac{\gamma p_{\mathrm{o}}}{(\gamma+1)^{2} P} \alpha^{3}$

Here $\alpha$ denotes the residual shock strength, which is related to the shock strength $M_{\mathrm{sh}}$ via

$M_{\mathrm{sh}}=\frac{U_{\mathrm{sh}}-u_{1}}{c_{\mathrm{S} 1}}=1+\alpha$

with $U_{\mathrm{sh}}$ as shock speed and $u_{1}$ and $c_{\mathrm{S} 1}$ as flow speed and sound speed in front of the shock (Landau \& Lifshitz 1987). Note that this definition deviates slightly from Eq. (5) because here we use $c_{\mathrm{S} 1}$ instead of $c_{\mathrm{T} 1}$ in the denominator (see Paper I). The limiting shock strength $\alpha_{\text {lim }}$ can be calculated if the generalized continuity Eq. (29) is applied to the case of acoustic waves following the derivation in Sect. 3. We then obtain

$\alpha_{\lim } \simeq \frac{c_{\mathrm{So}} P}{4}\left(\frac{1}{H_{\rho}}-\frac{3}{2} \frac{1}{H_{\mathrm{T}}}-\frac{1}{L}\right)$.

The limiting value $\eta_{\text {lim }}$ is given by

$\eta_{\mathrm{lim}} \simeq \frac{c_{\mathrm{So}} P}{\gamma+1}\left(\frac{1}{H_{\rho}}-\frac{3}{2} \frac{1}{H_{\mathrm{T}}}-\frac{1}{L}\right)$.

For the definition of $H_{\rho}, H_{\mathrm{T}}$, and $L$ see Eqs. (33), (32) and (36), respectively. In case of plane acoustic waves, we have $L^{-1}=0$. The general case of acoustic tube waves is analogical to longitudinal tube waves with the assumption $\mathcal{Z}=1$ (see Eq. (37)).

\section{Appendix B: Detailed solution for $\hat{\alpha}(z)$}

In Sect. 3, we derived an approximate expression for $\hat{\alpha}_{\text {lim }}$ valid under various restrictive assumptions. In order to obtain a general expression for $\hat{\alpha}_{\lim }$ as well as the solution for $\hat{\alpha}(z)$, the equation

$2 \frac{\mathrm{d} \hat{\alpha}}{\mathrm{d} z}+\left(\frac{3}{2} \frac{1}{H_{\mathrm{T}}}-\frac{1}{H_{\rho}}+\frac{1}{A} \frac{\mathrm{d} A}{\mathrm{~d} z}\right) \hat{\alpha}+\frac{4 \hat{\alpha}^{2}}{c_{\mathrm{So}} P \mathcal{Z}}=0$

needs to be solved, which constitutes a Riccati ODE of the form $y^{\prime}=f(x) y^{2}+g(x) y+h(x)$ (e.g., Murphy 1960; Zwillinger 1992). As we have $h(x)=0$, we have the special case of a Verhulst ODE (or Bernoulli ODE of the order 2), which can

\footnotetext{
${ }^{2}$ In Paper I, Appendix A, the factors $\rho_{1}, p_{1}$, and $T_{1}$ need to be omitted on the RHS of Eqs. (A.6)-(A.8), respectively.
}

easily be reduced to a linear ODE of first order by the substitution $y=1 / s$. For Eq. (B.1), we thus obtain

$\frac{\mathrm{d} s}{\mathrm{~d} z}+\frac{1}{2} W s=\frac{1}{2} K$

with

$W=\frac{1}{H_{\rho}}-\frac{3}{2} \frac{1}{H_{\mathrm{T}}}-\frac{1}{A} \frac{\mathrm{d} A}{\mathrm{~d} z}$

and

$K=\frac{4}{c_{\mathrm{So}} P \mathcal{Z}}$.

This equation can be solved in a straightforward manner with an integrating factor. With the substitutions

$W^{*}=\frac{1}{z} \int_{0}^{z} W\left(z^{\prime}\right) \mathrm{d} z^{\prime}$

and

$I=\frac{1}{2} W^{*} \mathrm{e}^{-\frac{1}{2} W^{*} z} \int_{0}^{z} \mathrm{e}^{\frac{1}{2} W^{*} z^{\prime}} \mathrm{d} z^{\prime}$

we obtain as general solution for $s(z)$

$s=\frac{K I}{W^{*}}+C \mathrm{e}^{-\frac{1}{2} W^{*} z}$

with $C$ as integration constant. With the backward substitution $s=1 / \hat{\alpha}$, we finally obtain

$\hat{\alpha}=\left[\frac{K I}{W^{*}}+\left(\frac{1}{\hat{\alpha}_{\mathrm{o}}}-\frac{K I}{W^{*}}\right) \mathrm{e}^{-\frac{1}{2} W^{*} z}\right]^{-1}$.

Here we determined the integration constant $C$ by taking $\hat{\alpha}=$ $\hat{\alpha}_{\mathrm{o}}$ for $z=0$ as endpoint condition. For large values of $z$ we obtain

$\hat{\alpha}_{\lim }=\frac{W^{*}}{K I}$.

In case that $W^{*}$ is essentially independent of $z$, we find $W^{*} \simeq W$ and $I \simeq 1$ which leads to

$\hat{\alpha}_{\lim } \simeq \frac{W}{K}$

in agreement with Eq. (37).

It should be noted that Eq. (B.9) is universially applicable contrary to Eq. (37) and also provides better numerical stability in some cases as, e.g., for $\hat{\alpha} \simeq 0$. In addition, Eq. (B.8) ensures that $\hat{\alpha} \geq 0$ as physically required. This requirement may be violated by Eq. (37) depending on $H_{\rho}, H_{\mathrm{T}}$, and $L$.

\section{References}

Cuntz, M. 1990, ApJ, 353, 255

Cuntz, M. 1999, A\&A, 350, 1100 (Paper I)

Cuntz, M., \& Ulmschneider, P. 1988, A\&A, 193, 119

Cuntz, M., Rammacher, W., \& Ulmschneider, P. 1994, ApJ, 432, 690

Cuntz, M., Ulmschneider, P., \& Musielak, Z. E. 1998, ApJ, 493, L117

Cuntz, M., Rammacher, W., Ulmschneider, P., Musielak, Z. E., \& Saar, S. H. 1999, ApJ, 522, 1053

Edwin, P. M., \& Roberts, B. 1983, Sol. Phys., 88, 179 
Fawzy, D. E., Ulmschneider, P., \& Cuntz, M. 1998, A\&A, 336, 1029

Fawzy, D., Rammacher, W., Ulmschneider, P., Musielak, Z. E., \& Stępień, K. 2002a, A\&A, 386, 971

Fawzy, D., Ulmschneider, P., Stȩpień, K., Musielak, Z. E., \& Rammacher, W. 2002b, A\&A, 386, 983

Herbold, G., Ulmschneider, P., Spruit, H. C., \& Rosner, R. 1985, A\&A, 145, 157

Landau, L. D., \& Lifshitz, E. M. 1987, Fluid Mechanics, Course of Theoretical Physics, 2nd Ed. (Oxford: Pergamon Press)

Mihalas, D., \& Mihalas, B. W. 1984, Foundations of Radiation Hydrodynamics (New York: Oxford University Press)

Murphy, G. M. 1960, Ordinary Differential Equations and Their Solutions (Princton: van Nordstrand Co.)

Musielak, Z. E. 2004, in Stars as Suns: Activity, Evolution, Planets, IAU Symp. 219, ed. A. Dupree, \& A. O. Benz, ASP Conf. Ser., in press

Musielak, Z. E., Rosner, R., Gail, H.-P., \& Ulmschneider, P. 1995, ApJ, 448, 865

Musielak, Z. E., Rosner, R., \& Ulmschneider, P. 2000, ApJ, 541, 410

Narain, U., \& Ulmschneider, P. 1996, Space Sci. Rev., 75, 453

Pozrikidis, C. 1997, Introduction to Theoretical and Computational Fluid Dynamics (New York: Oxford University Press)
Rae, I. C., \& Roberts, B. 1982, ApJ, 256, 761

Rammacher, W. 1991, Ph.D. Thesis, Univ. Heidelberg

Roberts, B. 1981, in Physics of Sunspots, ed. L. E. Cram, \& J. H. Thomas, Sacramento Peak Observatory, Sunspot, New Mexico, 369

Roberts, B. 1983, Sol. Phys., 87, 77

Solanki, S. K. 1996, in Stellar Surface Structure, ed. K. G. Strassmeier, \& J. L. Linsky (Dordrecht: Kluwer), IAU Symp., 176, 201

Spruit, H. C. 1981a, in The Sun as a Star, ed. S. Jordan, NASA, Washington, NASA SP-450, 385

Spruit, H. C. 1981b, A\&A, 98, 155

Stępień, K., \& Ulmschneider, P. 1989, A\&A, 216, 139

Ulmschneider, P. 1970, Sol. Phys., 12, 403

Ulmschneider, P., \& Musielak, Z. E. 1998, A\&A, 338, 311

Ulmschneider, P., \& Musielak, Z. E. 2003, in Current Theoretical Models and Future High Resolution Solar Observations, ed. A. A. Pevtsov, \& H. Uitenbroek, ASP Conf. Ser., 286, 363

Ulmschneider, P., Musielak, Z. E., \& Fawzy, D. E. 2001, A\&A, 374, 662

Zwillinger, D. 1992, Handbook of Differential Equations (Boston: Academic Press) 